Journal of Computer Science 4 (12): 1036-1041, 2008

ISSN 1549-3636

(C) 2008 Science Publications

\title{
Analysis of Robustness for Convex Optimization Applied to Array Antenna Pattern Synthesis
}

\author{
${ }^{1}$ Richard Torrealba, ${ }^{1}$ David H. Covarrubias and ${ }^{2}$ Marco Panduro \\ ${ }^{1}$ Department of Electronics and Telecommunication, CICESE Research Center, \\ Km. 107 Carretera Tijuana-Ensenada, Ensenada, BC, Mexico, C.P. 22860 \\ ${ }^{2}$ Reynosa-Rodhe Multidisciplinary Academic Center, Universidad Autonoma de Tamaulipas, \\ Carretera Reynosa-San Fernando, Reynosa, Tamaulipas, 88779 Mexico
}

\begin{abstract}
This study presents an analysis of the convex optimization applied to the synthesis of the radiation pattern for linear antenna arrays. This study emphasizes the application of the convex optimization for the array pattern synthesis considering the simultaneous elimination of several zones interferences, reduction of the level of power in two space zones densely populated by interferences, as well as the variation of these zones in terms of proximity-distance of the source of interest, variation of the size of the interferences zones and the number of zones within the radiation pattern. Simulation results are provided. These results define certain levels where the linear array could be exploited to achieve a maximum performance.
\end{abstract}

Key words: Convex optimization, antenna arrays, radiation pattern synthesis, mobile communications

\section{INTRODUCTION}

Mobile communications systems are facing an increasing demand for heterogeneous broadband services and applications. Given the limited spectrum available to provide high data rate communication for an increasing number of cellular subscribers, it is generally expected that the deployment of smart antennas will increase the overall system capacity and performance. Smart antennas will not only increase the antenna gain, but also reduce interference and delay spread by means of spatial filtering and thus enhance the properties of the mobile radio channel required for high data rate communication. The performance increase to be expected when using smart antennas highly depends on how accurate the interference pattern is reduced while maintaining a maximum gain in the direction of the signal of interest. Since early before the advent of mobile communications, adaptive array techniques aimed at reducing interference ${ }^{[1]}$. Various adaptive methods have quickly been established, evolving toward several applications ${ }^{[2]}$. Being directed toward either the signal processing or the antenna design, the application of smart arrays to mobile communications soon reached a boom, either involving space diversity methods, or advanced adaptive spatialtemporal receivers for spread-spectrum systems, e.g. ${ }^{[3]}$.
The antenna array pattern synthesis problem consists of finding weights that satisfy a set of specifications on the beam pattern. The array antenna pattern synthesis can be obtained applying different analytic techniques ${ }^{[1-3]}$ and in recent years techniques based on genetic algorithms ${ }^{[4]}$ and differential evolution $^{[5]}$. An important comment in ${ }^{[3]}$ is that in many minimization methods, there is no guarantee that we can reach the absolute optimum unless the problem is convex.

In the last two decades, several fundamental and practical results have been obtained in convex optimization theory ${ }^{[6,7]}$. The engineering community not only has benefited from these recent advances by finding applications but has also fuelled the mathematical development of both the theory and efficient algorithms. The two classical mathematical references on the subject are ${ }^{[8,9]}$.

The antenna array synthesis problem can often be expressed as a convex optimization problem which can be solved numerically with algorithms such as interior point methods ${ }^{[10]}$. Results reported by Lebret ${ }^{[10]}$, show that the problem of the minimization of the side lobe level of the radiation pattern can be solved by convex optimization. Lebret makes an analysis of the convex optimization to synthesize antenna arrays. However, this analysis is limited to simple design cases for the linear array pattern.

Corresponding Author: David H. Covarrubias, Department of Electronics and Telecommunication, CICESE Research Center, Km. 107 Carretera Tijuana-Ensenada, Ensenada, BC, Mexico, C.P. 22860

Tel: +(52-646) 175-05-00 Fax: +(52-646) 175-05-54 


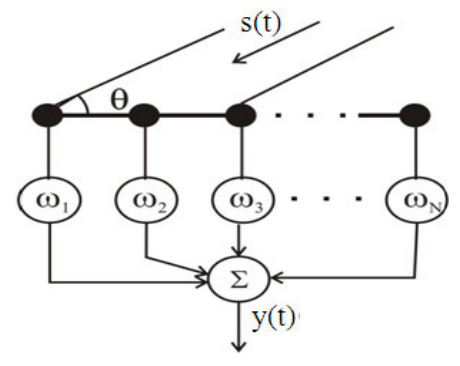

Fig. 1: Uniform Linear Array (ULA) of $\mathrm{N}$ equispaced identical elements

This study illustrates the application of the convex optimization for the array antenna pattern synthesis, considering the simultaneous elimination of several zones interferences, reduction of the level of power in two space zones densely populated by interferences, as well as the variation of these zones in terms of proximity-distance of the source of interest, variation of the size of the interferences zones and the number of zones within the radiation pattern.

The purpose of this study is to evaluate the limits of performance for linear antenna arrays and to define the levels and the cases where the linear array could be exploited to achieve a maximum performance.

Problem statement: An antenna array is a configuration of individual radiating elements that are arranged in space and can be used to produce a directional radiation pattern. We assume that in a mobile communications system, the base station is equipped with a Uniform Linear Array (ULA) of $\mathrm{N}$ equidistant identical elements (Fig. 1). The array receives the signals from the $\mathrm{K}$ users located in the far field zone of the array. We suppose all the signals to be uncorrelated and the waves are propagating in a homogeneous medium, this is, the unique spread effect, the source to the array is only the arrival time $\tau$. Let a desired signal from far field impinge on the array from a known Direction of Arrival (DOA) $\theta_{0}$ along with $\mathrm{M}$ uncorrelated interfering signals from unknown DOAs $\left\{\theta_{1}, \theta_{2}, \ldots, \theta_{\mathrm{M}}\right\}$, respectively.

The radiation pattern of the array depends on the configuration, the distance between the elements, the amplitude and phase excitation of the elements and also the radiation pattern of individual elements ${ }^{[1]}$. Since the array elements are identical, we can assume the radiation pattern of the array considering the sum of all contributions signals, $x(t)$, of each individual element:

$$
x(t)=s(t) \sum_{i=1}^{N} \omega_{i} e^{j 2 \pi f_{0} \tau_{i}}
$$

The above relation as often referred to as pattern multiplication, which indicates that the total field of the array is equal to the product of the field due to the single element located at the origin and a factor called array factor, $\mathrm{AF}$, defined as:

$$
\mathrm{AF}(\theta)=\sum_{\mathrm{n}=1}^{\mathrm{N}} \omega_{\mathrm{i}} \mathrm{e}^{\mathrm{jkx} x_{\mathrm{i}} \cos \theta}
$$

Where:

$\mathrm{k}=2 \pi / \lambda=$ The wave number and denotes the positions of each element in the array

$\omega=\left[\omega_{1} \ldots \omega_{\mathrm{N}}\right]=$ The weight vector, which is chosen to give a desired beam pattern

The synthesis of the antenna array pattern is a procedure in which an array of antennas is exploited to achieve maximum reception in a specified direction by estimating the signal of arrival from a desired direction (in the presence of noise) while signals of the same frequency from other directions are rejected. This is achieved by varying the weights across the array.

The output of array can be expressed as ${ }^{[12]}$

$$
y(t)=\omega^{T} x(t)
$$

Where:

$x(t)=$ The signal vectors received by the elements of array

$\omega^{\mathrm{T}}=$ The transpose vector of the weight vector $\omega=$ $\left[\omega_{1} \ldots \omega_{\mathrm{N}}\right]$

It is assumed that the signal incident on the array can be modeled as stationary stochastic processes. The mean output power of the array is given by:

$\mathrm{P}(\omega)=\omega^{\mathrm{T}} \mathrm{R} \omega$

in (4) R is the array correlation matrix defined by:

$R=E\left[x(t) x^{T}(t)\right]$

where, E denotes expected value. The response of the array to a plane wave of frequency $\mathrm{f}_{0}$ incident on the array from a direction $\theta$ can be represented as:

$\mathrm{r}(\theta)=\mathrm{C}(\theta) \omega$

$\mathrm{C}$ is the steering matrix ${ }^{[13]}$, which contain the contribution of $\mathrm{N}$ elements of array and is given by: 
$\mathrm{C}=\left[\begin{array}{l}\mathrm{D} \\ \mathrm{B}\end{array}\right]$

In this case, $\mathrm{D}$ and $\mathrm{B}$ denotes the real and imaginary part of the output of the array, respectively:

$$
\begin{aligned}
& \mathrm{D}=\cos \left(2 \pi \mathrm{f}_{0} \tau_{\mathrm{j}}(\theta)\right) \\
& \mathrm{B}=\operatorname{sen}\left(2 \pi \mathrm{f}_{0} \tau_{\mathrm{j}}(\theta)\right), \mathrm{j}=1, \ldots, \mathrm{N}
\end{aligned}
$$

$\tau_{\mathrm{j}}$ is the arrival time of the wave in the $\mathrm{j}$ element of the array.

The response of the array can be fixed in $\mathrm{K}$ directions by imposing a linear constraint of the following form on the weight vector:

$$
\mathrm{C} \omega=\mathrm{d}
$$

Where:

$$
\mathrm{C}=\left[\begin{array}{c}
\mathrm{C}\left(\theta_{1}\right) \\
\mathrm{C}\left(\theta_{2}\right) \\
\vdots \\
\mathrm{C}\left(\theta_{\mathrm{k}}\right)
\end{array}\right]_{\mathrm{k} \times \mathrm{N}}
$$

and

$$
d=\left[\begin{array}{c}
r\left(\theta_{1}\right) \\
r\left(\theta_{2}\right) \\
\vdots \\
r\left(\theta_{k}\right)
\end{array}\right]
$$

$\mathrm{d}$ specifies the desired response in each direction of $\left(\theta_{1}\right.$, $\left.\theta_{2}, \ldots, \theta_{\mathrm{k}}\right)$.

By using the previous notation we can formulate the synthesis of the antenna array pattern as the next optimization problem ${ }^{[12]}$ :

$$
\begin{array}{cr}
\min & \omega^{\mathrm{T}} \mathrm{R} \omega \\
\text { subject to } & \mathrm{C} \omega=\mathrm{d}
\end{array}
$$

Usually $\mathrm{C}$ and $\mathrm{d}$ are defined by the desired signal direction, which is assumed know. The optimization problem indicated by Eq. 12 can be express as a convex optimization problem ${ }^{[13]}$ :

$$
\begin{array}{cc}
\text { minimize } & \omega^{\mathrm{T}} \mathrm{R} \omega \\
\text { subject to } & |\mathrm{C} \omega-\mathrm{d}|+\Gamma|\omega| \leq \varepsilon
\end{array}
$$

where, $\Gamma \geq 0$ is a robustness matrix, is a tolerance vector.
For solving the problem denoted in (13) using convex optimization, is convenient to present some concepts to understand and apply convex optimization. For example:

- If a set $\mathrm{C}$ is convex for all $\mathrm{x}_{1}, \mathrm{x}_{2} \in \mathrm{C}$ and all real number $\alpha, 0<\alpha<1$, then the point $\alpha \mathrm{x}_{1}+(1-\alpha) \mathrm{x}_{2} \in \mathrm{C}$

- A function $\mathrm{f}$ is convex on a convex set if $f\left(\alpha x_{1}+\left(1-\alpha x_{2}\right)\right) \leq \alpha f\left(x_{1}\right)+(1-\alpha) f\left(x_{2}\right)$ for $\alpha: 0 \leq \alpha$

The convex optimization problems can be solved efficiently by Interior Point Method (IPM) ${ }^{[14,15]}$.

Examples of convex functions are: the affine function $\mathrm{a}^{\mathrm{T}} \mathrm{x}+\mathrm{b}$ where $\mathrm{a}, \mathrm{x}$ are vectors and $\mathrm{b}$ is a scalar, quadratics functions $x^{T} \mathrm{Rx}$ and norm of vectors $\|x\|$ (which include the Euclidian norm, the absolute value and the maximum value of a set of elements).

The convex optimization problems can be solved efficiently by Interior Point Method (IPM) ${ }^{[16,17]}$, so the problem (13) can be solved using IPM method. Then, it is necessary to express the problem (13) in the following way:

$$
\begin{array}{lc}
\text { minimize } & f^{T} x \\
\text { subject to } & \left\|A_{i} x+b_{i}\right\| \leq c^{T}{ }_{i} x+d_{i}, i=1, \ldots, m
\end{array}
$$

Where:

A $\quad=$ A positive semidefinite matrix in $\mathrm{R}^{2 \times(\mathrm{N}-1)}$

$\mathrm{b}, \mathrm{c}$ and $\mathrm{d}=$ vector in $\mathrm{R}^{2}$

$$
\mathrm{x}=\text { Optimization variable }
$$

\section{RESULTS AND DISCUSSION}

Simulation results for the pattern synthesis: When some information about the weights or the direction of arrival is only known approximately, it is essential that the performances of the array are not degraded with slightly different parameter values. To evaluate the convex optimization applied to the synthesis of the antenna array pattern, the scenarios proposed are mainly presented in terms of extremely adverse conditions, i.e., by increasing the isolation between main lobe and minors lobes at the radiation pattern; by rejecting interferences presented as individuals, as well as, grouping in $20^{\circ}$ wide zones of interferences; and by steering the main lobe at different users spatial positions. In the discussion we report four different experiments which were carried out to show the versatility of the convex optimization applied to the array synthesis. 
Beam pattern synthesis in broadside mode, three punctual interferences and two fixed size and wide zone angular of interferences: As a first goal in our simulations, we tried to optimize the beam pattern in the broadside mode, then, we consider an antenna array composed by 30 elements separated uniformly a distance $\lambda / 2$. The source of interest is located spatially in broadside mode $\left(90^{\circ}\right)$. Furthermore, we add as a constraint the reduction of the side lobe level at $-40 \mathrm{~dB}$, concerning to the two spaced zones densely populated by interferences (located at 62 and $118^{\circ}$ ) near the major lobe and the spaced zone width of $20^{\circ}$. Finally, we add to the evaluation the suppression of three punctual interferences located spatially at 20,40 and $140^{\circ}$. Figure 2 shows the radiation pattern already optimized. From Fig. 2, it is observed that when it is applied the convex optimization it is possible to reduce the level of the side lobes in the two angular zones, specified in a level of $-40 \mathrm{~dB}$ and simultaneously the cancellation of the three individual interferences. The side lobe level is reduced to $-19.05 \mathrm{~dB}$.

From this first scenario, we can conclude that the radiation pattern satisfy the established design conditions.

Analysis of robustness considering variation of number of zone angular of interferences: We take into account the same simulation considerations that in the previous case, but now we consider the variation of the number of interferences angular zones. Figure 3 shows the radiation pattern under the influence of this parameter.

From Fig. 3a, we can observe that for the zone angular of interferences ranked from $61-82^{\circ}$, the side lobe level maintain the $40 \mathrm{~dB}$ level of isolation, as well as the level of the rest of the side lobes maintain uniformly at $-26.01 \mathrm{~dB}$, below that settled down in the design considerations. The three established individual interferences are also canceled. In a same way, in the radiation pattern response of the Fig. $3 b$, two zone angular of interferences ranked from $61-82^{\circ}$ and from $98-118^{\circ}$, carried on the $40 \mathrm{~dB}$ isolation level, but now the level of the side lobes was increased in $26.7 \%$ ($19.01 \mathrm{~dB})$ remaining marginally above the recommended isolation level. The radiation patterns showed in Fig. 3c and d corresponds to three and four zone of interferences, respectively. In both cases the side lobe and isolation levels decrease and does not fulfill the design considerations, because of the zones angular of interferences are located at the opposite ends of the uniform linear array operation window. In all cases the three established individual interferences were correctly canceled.
From this experiment, we can conclude that the robustness for convex optimization taking into account the presence of angular zones of interferences is marginally affected and the array beam pattern can be adjusted in some issues like the width of the major lobe, isolation level or the side lobe level.

Analysis of robustness shifting the angular zones of interferences: We take into account the same simulation considerations that in the first case, but now we impose as a restriction moving away the two $20^{\circ}$ wide angular zones as regards to the major lobe. We consider initially two angular zones located in 63 and $97^{\circ}$, both far away $4^{\circ}$ of the major lobe. (Fig. 4a) shows the array beam pattern response, in which does not fulfill the $19 \mathrm{~dB}$ isolation level required but maintain the level of $-40 \mathrm{~dB}$ at the angular zones. distance of the angular zones to the main beam Increasing now the distance between angular zones of interferences as regards to the major lobe at $5.5^{\circ}, 10.5^{\circ}$ and so on we obtain the array beam pattern response showed at Fig. 4b, c and d. Particularly, Fig. 4d shows the behavior of the beam pattern response, in terms on the side level lobe, as we increase the mentioned distance. We can conclude that in order to keep the robustness for convex optimization, applied to the antenna array beam pattern synthesis problem, it is necessary that the interferences zones must be separated $5^{\circ}$ at least regarding the major lobe.

Analysis of robustness increasing the wide of the interferences angular zones: Now we impose as a restriction to increase the width of the two interferences zones, also, in a same way that in the previous simulations, we want to eliminate three punctual interferences, reduce and make uniform the side lobe level and we should keep the major lobe width in $5^{\circ}$.

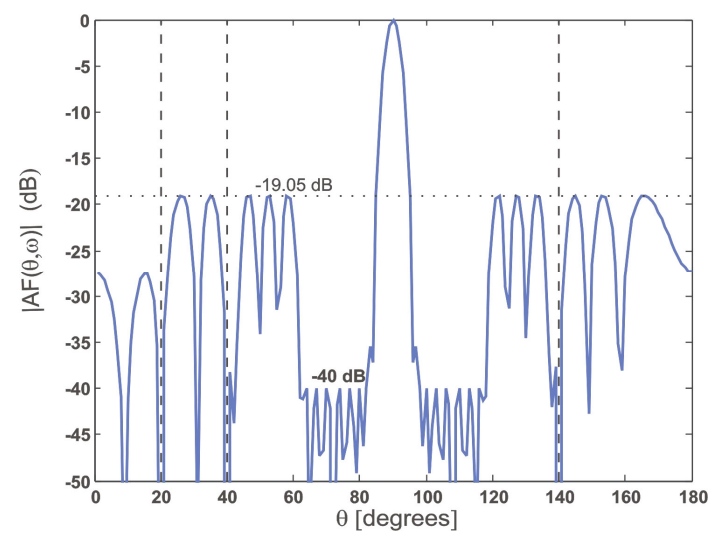

Fig. 2: Optimized diagram to reduce the level of lateral lobes in $-40 \mathrm{~dB}$ for two densely populated angular zones with interferences 


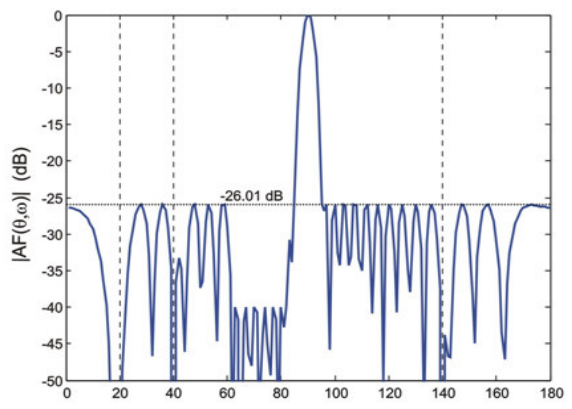

(a)

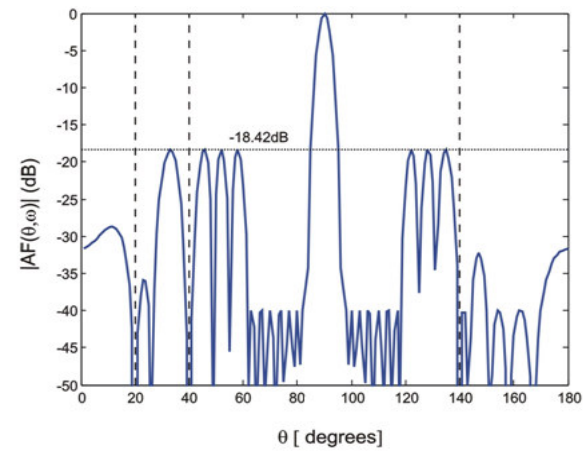

(c)

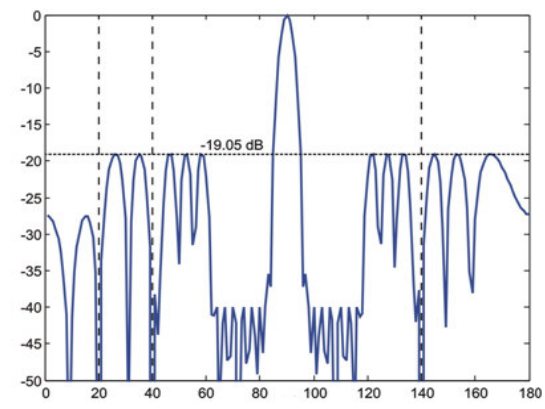

(b)

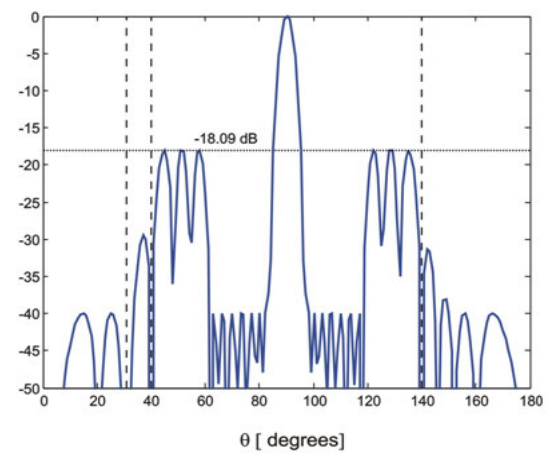

(d)

Fig. 3: Optimized pattern to reduce the side lobe level at $-40 \mathrm{~dB}$, in deferens space zones densely populated by interferences, with a space zones width of $20^{\circ}$. (a): One space zone; (b): Two space zones of interferences; (c): three spaces zones; (d): Four space zones of interferences

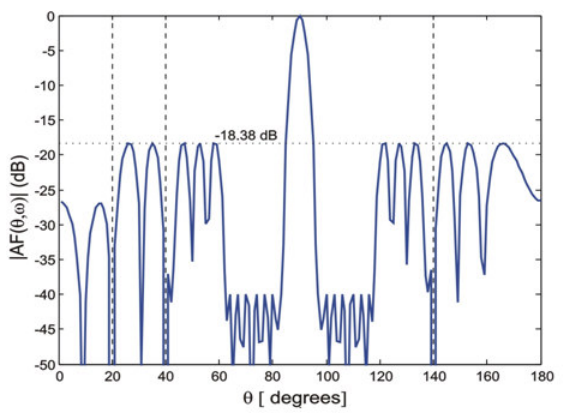

(a)

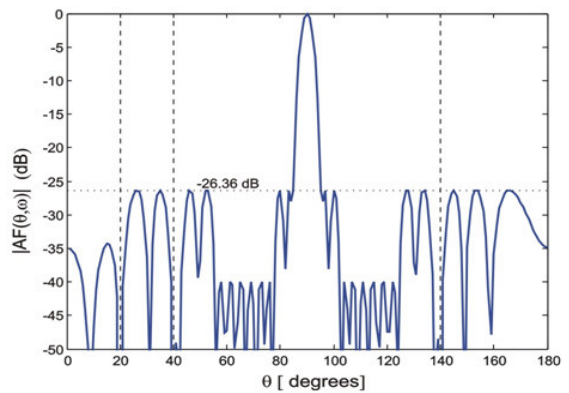

(c)

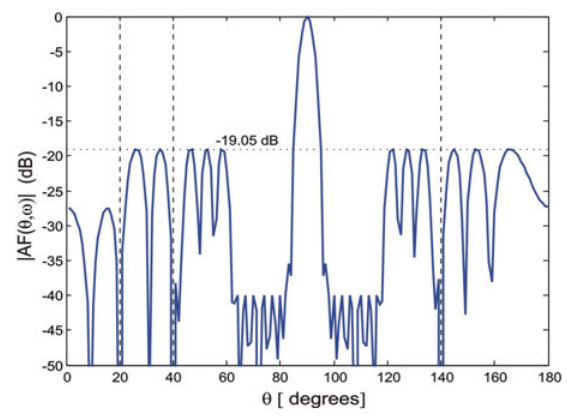

(b)

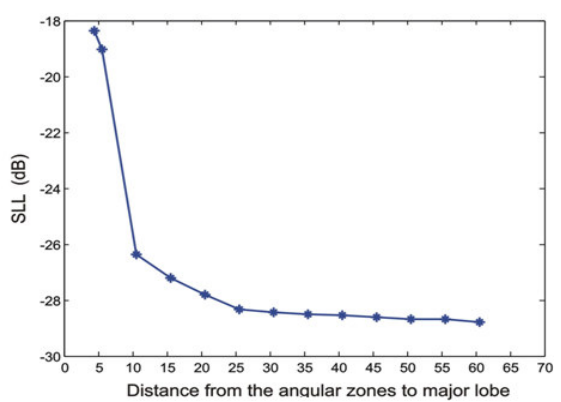

(d)

Fig. 4: Optimized diagrams to reduce the level of lateral lobes in $-40 \mathrm{~dB}$ in two densely populated zones with interferences, a wide zone one of $20^{\circ}$. In this case the zones move away of the main beam. (a): Separated zones $4^{\circ}$ of the main beam; (b): Separated zones 5.5 ; (c): Separated zones 10,5; (d): SLL with respect to them 


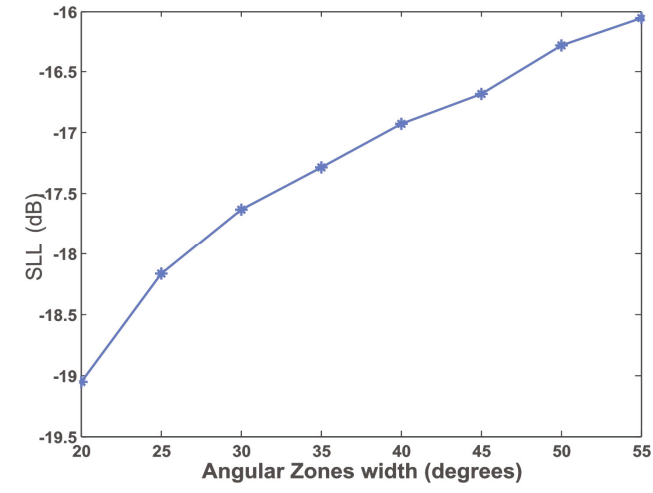

Fig. 5: SLL with respect to the wide one of the angular zones to the main beam

The two angular zones are located spatially at 62 and $98^{\circ}$ started both with $20^{\circ}$ width. Figure 5 shows the behavior of the side lobe level with different angular zones widths. This behavior shows particularly that the critical parameter in the analysis of robustness is precisely the wide of the interferences zones, affecting significantly the isolation level when the angular width is bigger than $20^{\circ}$.

\section{CONCLUSION}

In this study we emphasized in the application of the convex optimization for the array antenna pattern synthesis, considering the simultaneous elimination of several zones interferences, reduction of the level of power in two space zones densely populated by interferences, as well as the variation of these zones in terms of proximity-distance of the source of interest, variation of the size of the interferences zones and the number of zones within the radiation pattern. From the obtained results we found that the convex optimization is sensible to the distance to which are the zones of scatterers respect to the wide one of the main beam. With this one sets out to work in zones of interferences that are remote at least $5^{\circ}$ with respect to the main beam, thus to obtain diagrams that fulfil the design considerations

\section{ACKNOWLEDGEMENT}

This study was supported by the Mexican National Science and Technology Council, CONACyT, under grants J50839' Y and 52374.

\section{REFERENCES}

1. Schelkunoff, S.A., 1943. A mathematical theory of linear arrays. Bell. Syst. Tech. J., 22: 80-107.
2. Dolph, C.L., 1946. A current distribution for broadside arrays which optimizes the relationship between beam width and side-lobe level. Proc. IRE, 34: 335-348.

3. Bucci, O.M., D. D'Elia, G. Mazzarella and G. Panatiello, 1994. Antenna pattern synthesis: A new general approach. Proc. IEEE, 82: 358-371.

4. Panduro, M.A., D.H. Covarrubias, C.A.Brizuela and F.R. Marante, 2005. A multi-objective approach in the linear antenna array design. Int. J. Elect. Commun. (AEÜ), 59: 205-212.

5. Rocha, C., D.H. Covarrubias, C.A. Brizuela and M.A. Panduro, 2007. Differential evolution algorithm applied to sidelobe level reduction on a planar array. Int. J. Elect. Commun., (AEÜ), 61: 286-290.

6. Bertsekas, D.P., 1999. Nonlinear Programming. 2nd Edn., Athena Scientific, Belmont, MA.

7. Boyd, S. and L. Vandenberghe, 2004. Convex Optimization. 2nd Edn., Cambridge University Press, London.

8. Rockafellar, R.T., 1970. Convex Analysis. 2nd Edn., Princeton University Press, Princeton, New Jersey.

9. Luenberger, D.G., 1997. Optimization by Vector Space Methods. 1st Edn., Wiley, New York.

10. Lebret, H. and S. Boyd, 1997. Antenna array pattern synthesis via convex optimization. IEEE Trans Signal Process., 45: 526-532.

11. Van Veen, B.D. and K.M. Buckley, 1988. Beamforming: A versatile approach to spatial filtering. IEEE Acoust. Speech Signal Process. Mag., 5: 4-24

12. Evans, R.J. and K.M. Ahmed, 1984. An adaptive array processor with robustness and broad-band capabilities. IEEE Trans. Antennas Propagat., 32: 944-950.

13. Cantoni, A., X.G. Lin and K.L. Teo, 1993. A new approach to the optimization of robust antenna array processors. IEEE Trans. Antennas Propagat., 41: 403-411.

14. Hindi, H., 2006. A tutorial on convex optimization II: Duality and interior point methods. Proceeding of the Conference on American Control, June 1416, Minneapolis, Minnesota, pp: 11.

15. Nesterov, Y. and A. Nemirovsky, 1994. InteriorPoint Polynomial Methods in Convex programming. Vol. 13 of Studies in Applied Mathematics. SIAM, Philadelphia, PA.

16. Hindi, H., 2004. A tutorial on convex optimization. Proceeding of the Conference on American Control, Boston, Massachusetts. pp: 3252-3265.

17. Ahmed, K.M. and R.J. Evans, 1982, Robust adaptive array antennas. J. Acoust. Soc. Am., 71: 384-394.

18. Karmarkar, N., 1984. A new polynomial-time algorithm for linear programming. Combinatorica, 4: 373-395. 